University of Wollongong

Research Online

Faculty of Engineering and Information

Faculty of Engineering and Information

Sciences - Papers: Part A

Sciences

2011

A singularity free trajectory tracking method for the cooperative working of multi-arm robots using screw theory

Emre Sariyildiz

University of Wollongong, emre@uow.edu.au

Hakan Temeltas

Istanbul Technical University

Follow this and additional works at: https://ro.uow.edu.au/eispapers

Part of the Engineering Commons, and the Science and Technology Studies Commons

Research Online is the open access institutional repository for the University of Wollongong. For further information contact the UOW Library: research-pubs@uow.edu.au 


\title{
A singularity free trajectory tracking method for the cooperative working of multi- arm robots using screw theory
}

\author{
Abstract \\ In this paper we present a singularity free trajectory tracking method for the cooperative working of multi- \\ arm robot manipulators. It is based on an inverse kinematic transformation which determines the \\ manipulator's joint angles corresponding to the end-effector trajectory given in the task space. The \\ kinematic problem of multi-arm robot system is solved by using screw theory and quaternion algebra. \\ Screw theory is an effective way to establish a global description of rigid body and avoids singularities \\ due to the use of the local coordinates. Dual-quaternion is the most compact and efficient dual operator \\ to express screw displacement. Inverse kinematic solutions are obtained by using Paden-Kahan sub- \\ problems. The proposed method is implemented using two Stubli TX60 robot arms and simulation results \\ are given.

\section{Keywords} \\ multi-arm, working, cooperative, method, theory, tracking, screw, trajectory, free, singularity, robots \\ Disciplines \\ Engineering | Science and Technology Studies

\section{Publication Details} \\ Sariyildiz, E. \& Temeltas, H. (2011). A singularity free trajectory tracking method for the cooperative \\ working of multi-arm robots using screw theory. 2011 IEEE International Conference on Mechatronics, \\ ICM 2011 - Proceedings (pp. 451-456). United States: IEEE.
}




\title{
A Singularity Free Trajectory Tracking Method for the Cooperative Working of Multi-Arm Robots Using Screw Theory
}

\author{
Emre SARIYILDIZ $^{\# 1}$, Hakan TEMELTAS ${ }^{\# 2}$ \\ Department of Control Engineering, Istanbul Technical University \\ 34469 Istanbul, Turkey \\ ${ }^{1}$ esariyildizditu.edu.tr \\ ${ }^{2}$ hakan.temeltaseitu.edu.tr
}

\begin{abstract}
In this paper we present a singularity free trajectory tracking method for the cooperative working of multi-arm robot manipulators. It is based on an inverse kinematic transformation which determines the manipulator's joint angles corresponding to the end-effector trajectory given in the task space. The kinematic problem of multi-arm robot system is solved by using screw theory and quaternion algebra. Screw theory is an effective way to establish a global description of rigid body and avoids singularities due to the use of the local coordinates. Dualquaternion is the most compact and efficient dual operator to express screw displacement. Inverse kinematic solutions are obtained by using Paden-Kahan sub-problems. The proposed method is implemented using two Stäubli TX60 robot arms and simulation results are given.
\end{abstract}

Keywords-Cooperative working, Dual-Quaternion, MultiRobot Arm, Screw Theory, Singularity-Free Inverse Kinematic

\section{INTRODUCTION}

Single-arm robot systems have limited manipulation ability and versatility [1], [2]. For instance, single-arm industrial robots can't perform their roles in the many fields in which operators do the job with their two arms [3]. And also singlearm robot systems can't manipulate bulky objects whose weight exceeds the working capacities of the robot manipulator [4]. However cooperative working of multi-arm robot systems can solve these problems. In these reasons, the needs for multi-arm robot manipulator are increasing in many industrial fields [3]. In addition to industrial robotics, medical robotics and humanoid robotics are also the main application areas of cooperative working of multi-arm robot systems [5], [6].

The main problem of cooperative manipulation is to find out the appropriate way to control the system of robots and object in the work space at any stage of cooperative work. The first crucial problem is kinematic uncertainty to solve this problem [7]. In cooperative working of multi-arm robot systems, a closed chain mechanism comes into existence from open chain mechanisms [8]. In general, closed-chain mechanism's kinematic problems are more difficult than open chain mechanism. However in cooperative working of multiarm robot system, the kinematic problem of closed-chain robot arms can be solved by reducing the full kinematic problem into appropriate sub-open-chain kinematic problem. If all robot arms position and orientation can be determined appropriately, closed-chain robot kinematic problem can be reduced into a serial robot arm kinematic problem. Then it can be easily solved by using the kinematic equations of serial robot arm. Several methods are used to solve kinematic problem of multi-arm robot systems [4], [9], [10]. These methods are based on differential kinematics and D-H convention which is the most common method in robot kinematics. Differential kinematics based solutions suffer from singularity problem and errors due to both long-term numerical integration drift and incorrect initial joint angles. D-H convention based solution methods also suffer from singularity problem [18-19]. Another main method in robot kinematic is screw theory which is based on line transformations approach. In screw theory every transformation of a rigid body or a coordinate system with respect to a reference coordinate system can be expressed by a screw displacement, which is a translation by along a $\lambda$ axis with a rotation by a $\theta$ angle about the same axis [11]. This description of transformation is the basis of the screw theory. There are two main advantages of using screw, theory for describing rigid body kinematics. The first one is that it allows a global description of rigid body motion that does not suffer from singularities due to the use of local coordinates. The second one is that the screw theory provides a geometric description of rigid motion which greatly simplifies the analysis of mechanisms [12]. Several application of screw theory has been introduced in kinematic. Yang and Freudenstein were the first to apply line transformation operator mechanism by using the dual-quaternion as the transformation operator [13]. Yang also investigated the kinematics of special five bar linkages using dual $3 \times 3$ matrices [14]. This transformation operator has 18 parameters while just 6 parameters are needed. M. Murray solved 3-DOF and 6-DOF serial arm kinematics by using screw theory with $4 \times 4$ matrix operator [15]. This transformation operator needs 16 parameters while just 6 parameters are needed for description of rigid body motion. J. Funda analyzed transformation operators of screw motion and he found that dual operators are the best way to describe screw motion and also the dual-quaternion is the most compact and efficient dual operator to express screw displacement [16-17]. Finally E. Sariyildiz and H. Temeltas investigated the kinematic of 6dof serial robot arms by using screw theory with quaternion and dual-quaternion operators [18-19]. 
In this paper, a singularity free trajectory tracking method for the cooperative working of multi-arm robot manipulators is proposed. The proposed method is based on screw theory and it uses dual-quaternion which is the most compact and efficient dual operator to express screw displacement as a screw motion operator. Thus, the proposed method provides a singularity free and computationally efficient trajectory tracking solution for multi-arm robot manipulators. This paper is included the mathematical preliminary for line transformation and quaternion algebra in section 2, screw theory by using quaternion algebra in section 3 , the kinematic scheme of n-dof serial robot manipulator in section 4, cooperative working of dual-arm robots in section 5, simulation results of trajectory tracking application for cooperative working of dual-arm robot manipulator in section 6. Conclusions and future works are drawn in the final section.

\section{MAthematical PRELIMINARY}

\section{A. Line Geometry and Dual-Numbers}

A line can be completely defined by the ordered set of two vectors. First one is point vector $(\boldsymbol{p})$ which indicates the position of an arbitrary point on line, and the other vector is free direction vector $(\boldsymbol{d})$ which gives the line direction. A line can be expressed as

$$
L(\boldsymbol{p}, \boldsymbol{d})
$$

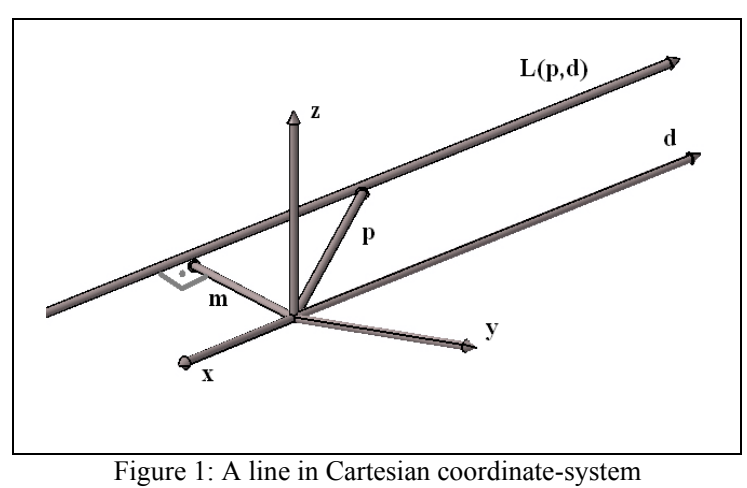

The representation $L(\boldsymbol{p}, \boldsymbol{d})$ is not minimal, because it uses six parameters for only four degrees of freedom.

\section{Plücker Coordinates}

An alternative line representation was introduced by $\mathrm{A}$. Cayley and J. Plücker. Finally this representation named after Plücker [20]. Plücker coordinates can be expressed as,

$$
\begin{aligned}
& L_{p}(\boldsymbol{m}, \boldsymbol{d}) \\
& \boldsymbol{m}=\boldsymbol{p} \times \boldsymbol{d}
\end{aligned}
$$

Both $\boldsymbol{d}$ and $\boldsymbol{m}$ are free vectors: $\boldsymbol{d}$ and $\boldsymbol{p}$ have the same meaning as before (they represent the direction of the line and the position of an arbitrary point on the line respectively) and $\boldsymbol{m}$ is the moment of $\boldsymbol{d}$ about the chosen reference origin. Note that $\boldsymbol{m}$ is independent of which point $\boldsymbol{p}$ on the line is chosen and the two three-vectors $\boldsymbol{d}$ and $\boldsymbol{m}$ are always orthogonal.

$$
\boldsymbol{p} \times \boldsymbol{d}=(\boldsymbol{p}+t \boldsymbol{d}) x \boldsymbol{d}
$$

$$
\boldsymbol{d} . \mathrm{m}=\mathbf{0}
$$

Plücker coordinates representation is also not minimal representation. The advantage of Plücker coordinate representation is that it is homogeneous: $L_{p}(\boldsymbol{m}, \boldsymbol{d})$ represents same line as $L_{p}(k \boldsymbol{m}, \mathrm{k} \boldsymbol{d})$, where $k \in \mathcal{R}$

\section{Intersection of Lines}

Let's define two lines in Plücker coordinates given by

$$
L a=(\boldsymbol{m a}, \boldsymbol{d a}) \text { and } L b=(\boldsymbol{m} \boldsymbol{b}, \boldsymbol{d} \boldsymbol{b})
$$

The intersection point of two intersection lines can be expressed as

$$
\begin{aligned}
& r=d b \times m b+(d a \times m a \cdot d b) \cdot d b \quad \text { or } \\
& r=d a \times m a+(d b \times m b \cdot d a) \cdot d a
\end{aligned}
$$

where $\boldsymbol{r}$ indicates the intersection point.

\section{Dual Numbers}

In analogy with a complex number a dual number can be defined as:

$$
\hat{u}=u+\epsilon u^{0}
$$

where $u$ and $u^{0}$ are real number and $\epsilon^{2}=0$. Dual numbers can be used to express Plücker coordinates. Let's define the orientation vector $(\boldsymbol{u})$ and the moment vector $\left(\boldsymbol{u}^{0}\right)$ given by

$\boldsymbol{u}=\left(x_{2}-x_{1}, y_{2}-y_{1}, z_{2}-z_{1}\right)$ and $\boldsymbol{u}^{0}=\boldsymbol{p} \times \boldsymbol{u}$

Then, the line which is defined in Plücker coordinates using dual numbers can be expressed as

$$
\begin{gathered}
L\left(\boldsymbol{u}^{0}, \boldsymbol{u}\right)=\boldsymbol{u}+\epsilon \boldsymbol{u}^{0} \\
\text { Quaternion \& Dual-Quaternion }
\end{gathered}
$$

Quaternions are hyper-complex numbers of rank 4, constituting a four dimensional vector space over the field of real numbers [21]. A quaternion can be represented as

$$
q=\left(q_{0}, \boldsymbol{q}_{v}\right)
$$

where $q_{0}$ is a scalar and $\boldsymbol{q}_{\boldsymbol{v}}=\left(q_{1}, q_{2}, q_{3}\right)$ is a vector. A quaternion with $\boldsymbol{q}_{\boldsymbol{v}}=\mathbf{0}$, is called as a real quaternion, and a quaternion with $q_{0}=0$, is called as a pure quaternion (or vector quaternion). Addition and multiplication of two quaternions can be expressed as

$$
q_{a}+q_{b}=\left(q_{a 0}+q_{b 0}\right),\left(\boldsymbol{q}_{a v}+\boldsymbol{q}_{b v}\right)
$$

$q_{a} \otimes q_{b}=q_{a 0} q_{b 0}-\boldsymbol{q}_{\boldsymbol{a v}} \boldsymbol{q}_{\boldsymbol{b v}}, q_{a 0} \boldsymbol{q}_{\boldsymbol{b} v}+q_{b 0} \boldsymbol{q}_{\boldsymbol{a} v}+\boldsymbol{q}_{\boldsymbol{a} v} \times \boldsymbol{q}_{\boldsymbol{b} v}$

where “®”, ".,, "x" denote quaternion product, dot product and cross product respectively. Conjugate and norm of the quaternion can be expressed as

$$
\begin{aligned}
& q^{*}=\left(q_{0},-\boldsymbol{q}_{v}\right)=\left(q_{0},-q_{1}-, q_{2},-q_{3}\right) \\
& \|q\|^{2}=q \otimes q^{*}=q_{0}^{2}+q_{1}^{2}+q_{2}^{2}+q_{3}^{2}
\end{aligned}
$$

When $\|q\|^{2}=1$, we get a unit quaternion. The inverse of a quaternion can be expressed as 


$$
q^{-1}=\frac{1}{\|q\|^{2}} q^{*} \quad \text { and } \quad\|q\| \neq 0
$$

that satisfies the relation $q^{-1} \otimes q=q \otimes q^{-1}=1$. For a unitquaternion we have $q^{-1}=q^{*}$. Unit quaternion can be defined as a rotation operator [22]. Rotation about a unit axis $\boldsymbol{n}$ with an angle $\theta$ can be expressed as

$$
q=\left(\cos \left(\frac{\theta}{2}\right), \sin \left(\frac{\theta}{2}\right) n\right)
$$

A dual-quaternion can be defined as

$$
\hat{q}=\left(\hat{q}_{o}, \widehat{\boldsymbol{q}}_{v}\right) \quad \text { or } \hat{q}=q+\epsilon q^{0}
$$

where $\hat{q}_{0}=q_{0}+\varepsilon q_{0}^{0}$, is a dual scalar, $\widehat{\boldsymbol{q}}_{\boldsymbol{v}}=\boldsymbol{q}_{\boldsymbol{v}}+\varepsilon \boldsymbol{q}_{\boldsymbol{v}}^{\mathbf{0}}$, is a dual vector, $q$ and $q^{0}$ are both quaternions, $\epsilon$ is the dual factor. Addition and multiplication of two dual-quaternions can be expressed as

$$
\begin{aligned}
& \hat{q}_{a}+\hat{q}_{b}=\left(q_{a}+q_{b}\right)+\epsilon\left(q_{a}^{0}+q_{b}^{0}\right) \\
& \hat{q}_{a} \odot \hat{q}_{b}=\left(q_{a} \otimes q_{b}\right)+\epsilon\left(q_{a} \otimes q_{b}^{0}+q_{a}^{0} \otimes q_{b}\right)
\end{aligned}
$$

where “ $\otimes$ " and " $\odot$ " denote quaternion and dual-quaternion product respectively. Conjugate, norm and inverse of the dual-quaternion are similar with quaternion. They can be expressed as

$$
\begin{gathered}
\hat{q}^{*}=q^{*}+\varepsilon\left(q^{0}\right)^{*} \\
\|\hat{q}\|^{2}=\hat{q} \odot \hat{q}^{*} \\
\hat{q}^{-1}=\frac{1}{\|\hat{q}\|^{2}} \hat{q}^{*}
\end{gathered}
$$

When $\|\hat{q}\|^{2}=1$, we get a unit dual-quaternion. For unit dual-quaternion these equations can be written given by

$$
\begin{gathered}
\|\hat{q}\|^{2}=\hat{q} \odot \hat{q}^{*}=1 \quad \text { and } \\
q \otimes q^{*}=1 \quad, q^{*} \otimes q^{0}+\left(q^{0}\right)^{*} \otimes q=0
\end{gathered}
$$

We can use unit dual-quaternion for general rigid transformation [18]. This transformation is very similar with pure rotation; however, not for a point but for a line. A line in Plücker coordinates $\left(L_{p}(\boldsymbol{m}, \boldsymbol{d})\right)$ can be expressed by using dual quaternions given by

$$
\hat{l}_{a}=l_{a}+\varepsilon m_{a}
$$

After transformation of $\hat{l}_{a}$ (rotation and t: translation) we obtain a transformed line $\hat{l}_{b}\left(\hat{l}_{b}=l_{b}+\varepsilon m_{b}\right)$. Transformation of a line can be expressed as,

$l_{b}=q \otimes l_{a} \otimes q^{*}$

$$
m_{b}=q \otimes m_{a} \otimes q^{*}+\frac{1}{2}\left(q \otimes l_{a} \otimes q^{*} \otimes t^{*}+t \otimes q \otimes l_{a} \otimes q^{*}\right)
$$

If we define a new quaternion $q^{\prime}=\frac{1}{2} t \otimes q$ ( $\mathrm{t}$ is translation) and a new dual quaternion $\hat{q}=q+\varepsilon q^{\prime}$, we can express the transformation of a line given by

$l_{b}+\varepsilon m_{b}=\left(q+\varepsilon q^{\prime}\right) \odot\left(l_{a}+\varepsilon m_{a}\right) \odot\left(q^{*}+\varepsilon q^{\prime *}\right)$

\section{SCREW THEORY}

The elements of screw theory can be traced to the work of Chasles and Poinsot in the early 1800s. According to Chasles all proper rigid body motions in 3-dimensional space, with the exception of pure translation, are equivalent to a screw motion (see fig. 2), that is, a rotation about a line together with a translation along the line [23].

The general screw motion operator can be represented by using dual-quaternion as follows:

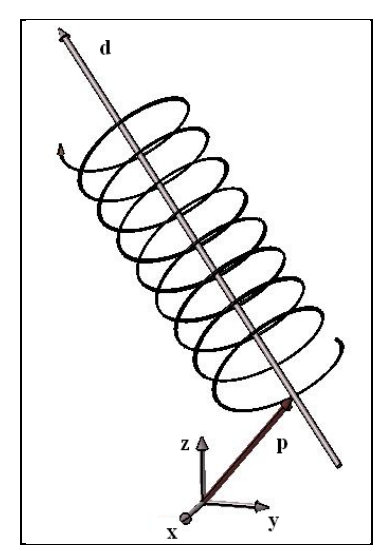

Figure 2: General screw motion

$$
\hat{q}=\cos \left(\frac{\widehat{\theta}}{2}\right)+\sin \left(\frac{\widehat{\theta}}{2}\right) \hat{d}
$$

where $\hat{\theta}=\theta+\varepsilon k$ and $\hat{d}=d+\varepsilon m$ are dual numbers. Here, $\theta$ and $d=[0, \boldsymbol{d}]$ indicate the rotation angle and the screw motion axis respectively. $m=[0, \boldsymbol{p} \times \boldsymbol{d}]$ indicates the moment vector of rotation axis where $\boldsymbol{p}$ is any point on the direction vector of $\boldsymbol{d}$ and $k=\boldsymbol{d}$.t. Further details of general screw motion formulation by using dual-quaternion can be found in [24].

\section{Manipulator Kinematic}

\section{A. Forward Kinematic}

1. Determining joint axis vector and moment vector: First we attach an axis vector which describes the motion of the joint. Then the moment vector of this axis is obtained for revolute joints by using the equation (3). Hence we obtain the Plücker notation of this axis.

2. Obtaining transformation operator: For all joints we obtain dual-quaternion for transformation operator as follows:

$\hat{q}_{i}=\left(\hat{q}_{0 i}, \hat{q}_{v i}\right) \quad$ and $\quad \hat{q}_{i}=q_{i}+\epsilon q_{i}^{0}$

where

$q_{i}=\cos \left(\frac{\theta_{i}}{2}\right)+\sin \left(\frac{\theta_{i}}{2}\right) \boldsymbol{d}_{i}$

$q_{i}^{0}=\frac{1}{2}\left(p_{i}-q_{i} \otimes p_{i} \otimes q_{i}\right) \otimes q_{i} \quad$ or $\quad q_{i}^{0}=\left[0, \sin \left(\frac{\theta_{i}}{2}\right) \boldsymbol{m}_{i}\right]$

where $\mathrm{i}=1,2 \ldots \ldots$. 
3. Formulation of rigid motion: Using (18) we can write multiplication of the dual-quaternion for n-DOF robot manipulator as follows:

$\hat{q}_{1 n}=\hat{q}_{1} \odot \hat{q}_{2} \odot \ldots . \odot \hat{q}_{n}$

Rotation and translation can be written as follows:

$R=q_{1 n} \otimes l_{n} \otimes q_{1 n}^{*}$

$T=q_{1 n} \otimes l_{n} \otimes q_{1 n}^{0}+q_{1 n} \otimes m_{n} \otimes q_{1 n}^{*}+q_{1 n}^{0} \otimes l_{n} \otimes q_{1 n}^{*}$

Using the equation (7), the position of the end effector can be expressed as

$\boldsymbol{t}=\left(\boldsymbol{q}_{v n} \times \boldsymbol{q}_{v n}^{\mathbf{0}}\right)+\left(\boldsymbol{q}_{v n-1} \times \boldsymbol{q}_{v n-1}^{0}\right) \cdot \boldsymbol{q}_{v n} * \boldsymbol{q}_{v n}$

where " $\times$ ", "." are cross and dot product respectively.

\section{B. Inverse Kinematic}

Paden-Kahan subproblems are used to obtain the inverse kinematic solution of robot manipulators. There are some main Paden-Kahan subproblems and also new extended subproblems [15], [25], [26]. We will use just three of them which occur frequently in inverse solutions for common manipulator design. To solve the inverse kinematics problem, we reduce the full inverse kinematics problem into appropriate subproblems. Solution of the inverse kinematics of serial robot arm can be seen in [18].

\section{COOPERATIVE Working OF Two SERIAL Robot ARMS}

Figure 3 illustrates a possible arrangement of two robot arms. For non-cooperative working, both robot arms forward and inverse kinematic solutions can be obtained independently by using above equations. In cooperative working of two robot arms, a closed chain mechanism comes into existence from two open chain mechanisms. As it shown in fig. 3 there are 12-dof in closed chain mechanism and there are infinite inverse kinematic solutions because of redundancy. Thus, closed-chain mechanism kinematic problems are more difficult then open chain mechanism in cooperative working. The kinematic problem of closed-chain robot arms can be solved by reducing the full kinematic problem into appropriate sub-open-chain kinematic problem. In fig. 3 a closed chain mechanism combines two serial-robot arms. If both robot arms position and orientation can be determined appropriately, closed-chain robot kinematic problem can be reduced into a serial robot arm kinematic problem. Then it can be solved more easily by using serialarm's kinematic solutions.

In this paper, two different methods are used to determine both of the robot arms' trajectories. In the first one, a path is determined for an object (ball). Then appropriate positions and orientations of two robot arms determined for each step of cooperative working. Inverse kinematic solutions are obtained for each step and cooperative working is done. In the second one, a path is determined for the first robot arm. Then the second robot arm path is determined by using first robot arm's position and orientation. Point symmetry method is used to obtain position and orientation of the second robot arm. The point symmetry method can be formulated as follows:
Let $q_{1}=\left(q_{10}, \boldsymbol{q}_{\mathbf{1} v}\right)$ and $q_{1}^{0}=\left(0, \boldsymbol{q}_{\mathbf{1} v}^{\mathbf{0}}\right)$ be the first robot arm's orientation and position informations. Then we can write position and orientation of the first robot arm in dualquaternion form given by

$\hat{q}_{1}=q_{1}+\epsilon q_{1}^{0}$

The second robot arm position and orientation can be defined by using point symmetry given by

$q_{2}=\left(q_{10},-q_{1 v}\right)$

$q_{2}^{0}=\left(0, \boldsymbol{q}_{1 v}^{0}+\lambda\right)$

where $\lambda$ is the distance vector between the first and the second robot arms' end effectors. In this case the first robot arm works as a master and the second robot arm works as a slave.

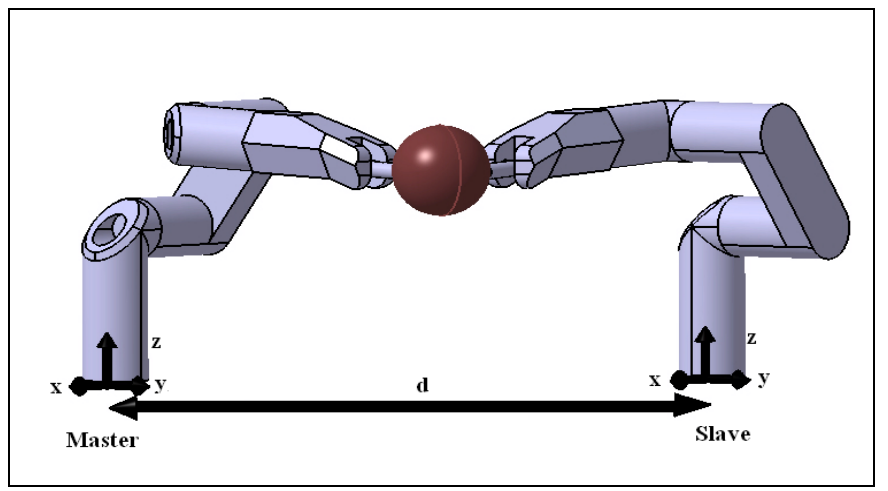

Figure 3: Configuration of dual-arm robot manipulator

\section{Simulation Results}

Simulation studies are made for Staubli TX60 serial robot manipulator. This series robots feature an articulated arm with 6 degrees of freedom for high flexibility. Matlab is chosen for simulations and animation applications are performed by using virtual reality toolbox of Matlab. Also Staubli TX60 iges file which can be freely obtained from Staubli's web page is used for animation application.

Two animation applications which are shown in figures 4 and 5 are performed. In the first one two robot arms carry a ball from its initial position to the desired target position as shown consecutively in figure 4 . To implement this case, first a path is determined for the ball. Then the inverse kinematic of serial robot arm is solved by using this path for both of the robot arms. In the second case, a master-slave mode working is performed. The first robot arm which has a ball at the end effector moves by a given path and the second robot arm follows the tip point of the first robot arm as shown consecutively in figure 5. To implement this case, first a path is determined for the first robot arm. Then the orientation and the position data of the first robot arm is sent to the second robot arm and inverse kinematics of second robot arm is solved by using these data. The first robot arm which sends its position and orientation data works as a master and the second robot arm which follows the tip point of the first robot arm works as a slave. 


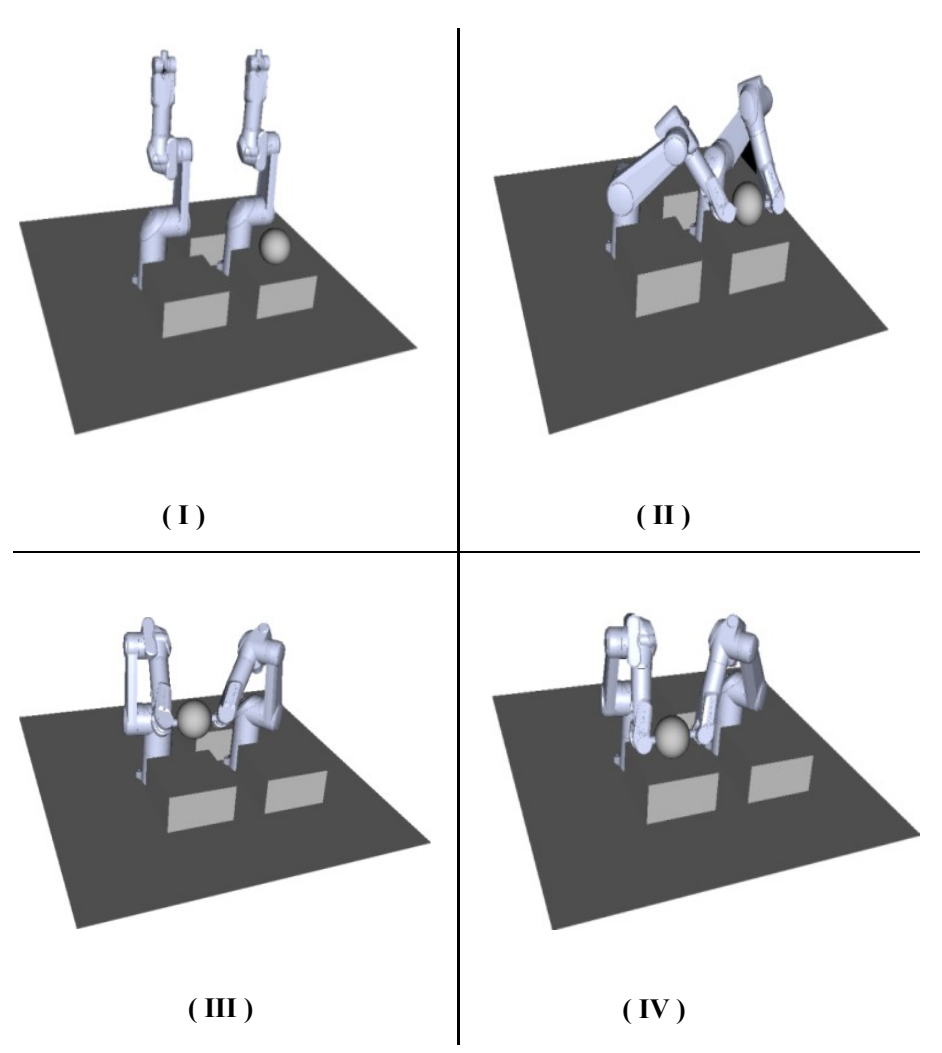

Figure 4: Cooperative working (carrying ball experiments)

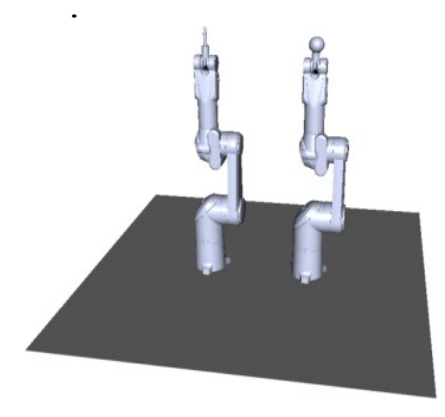

( I )

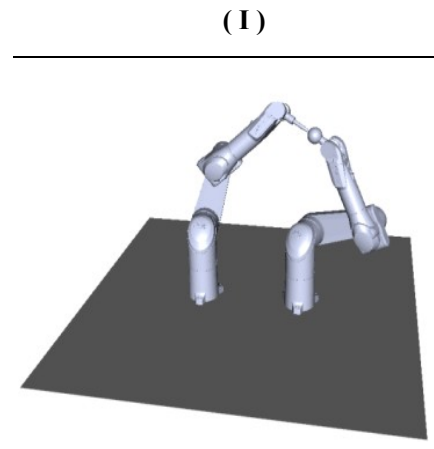

( III )

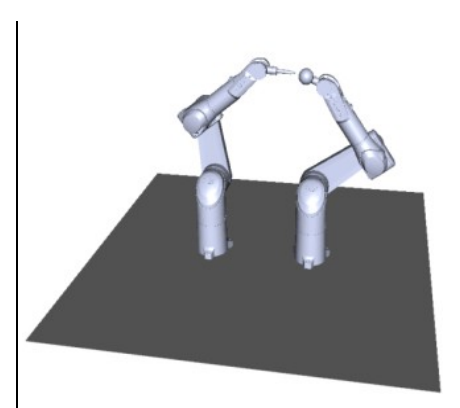

( II )

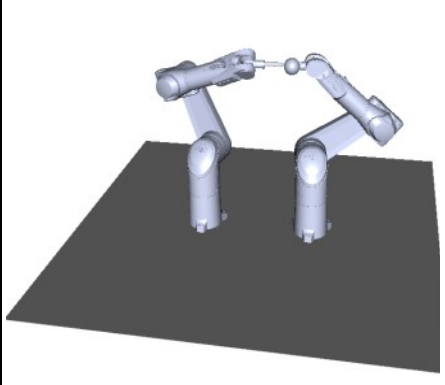

( IV )

Figure 5: Cooperative working (master slave mode working)

The dual-quaternion is the most compact and computationally efficient dual operator to express screw displacement. Here a comparative study is given for dualquaternion and homogenous transformation matrix based solutions. Figure 6 shows the total number of calculation of rigid transformation for dual-quaternion and homogenous transformation matrix. Figures 7 and 8 show the computation time of the forward and inverse kinematic algorithms. The computation time is evaluated using Matlab's tic-toc commands on a Core 2 Duo $2.2 \mathrm{GHz}$ PC with 2 GB RAM

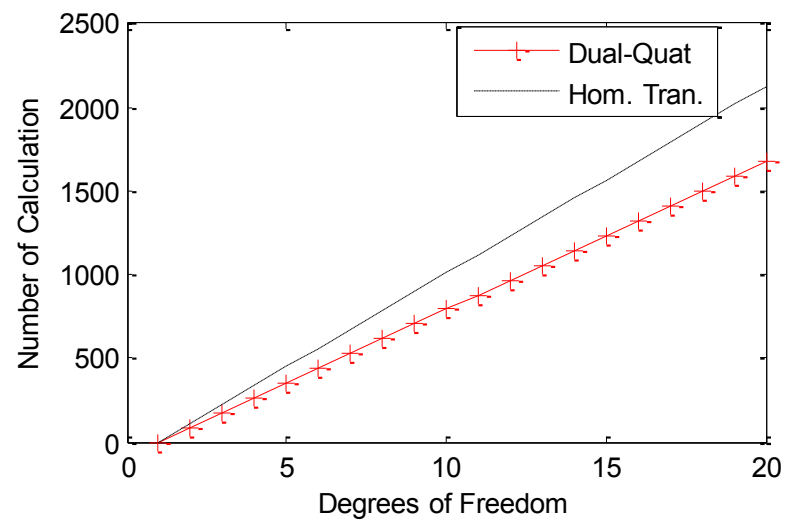

Figure 6: Performance comparison of the rigid body transformation chaining operations

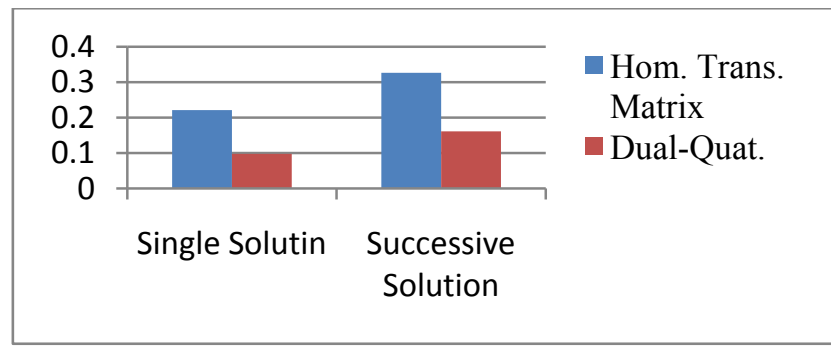

Figure 7: Relative simulation times of the forward kinematic solutions (second)

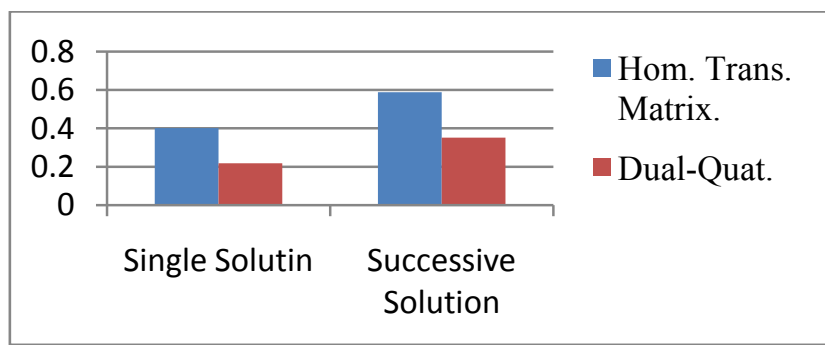

Figure 8: Relative simulation times of the inverse kinematic solutions (second)

In order to verify the trajectory tracking method we made a trajectory tracking application. Firstly, a circular trajectory is determined on the $\mathrm{x}, \mathrm{z}$ coordinates plane for the master robot arm. Then, the slave robot's trajectory is determined by using master robot's trajectory and the point symmetry method. Trajectory tracking results are shown in figures 9 and 10. As it can be seen from the Figures 9 and 10, a satisfactory singularity free trajectory tracking application is implemented. 


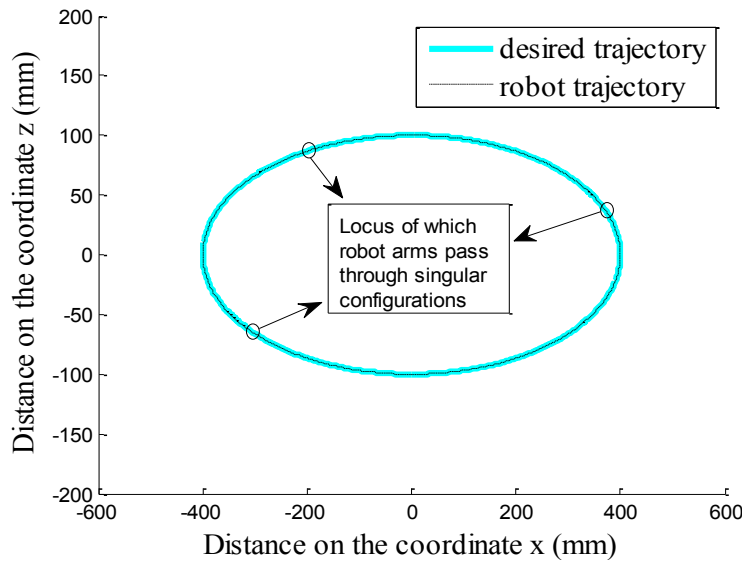

Figure 9: Trajectory tracking on the coordinates $\mathrm{x}$ and $\mathrm{z}$
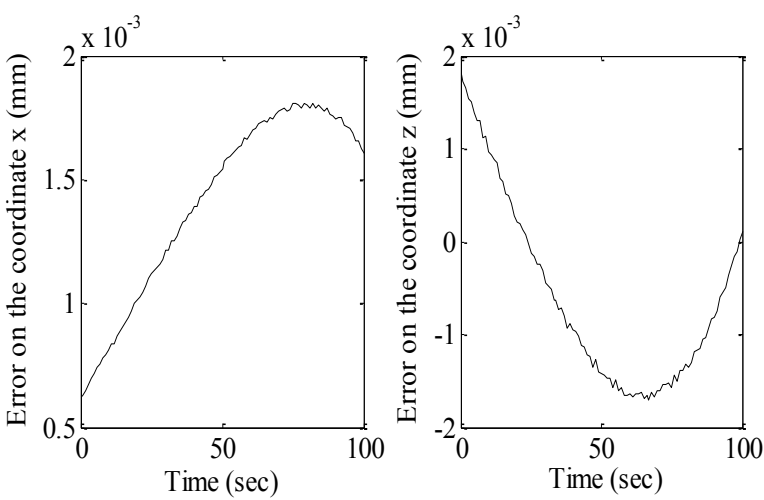

Figure 10: Trajectory tracking errors on the coordinates $\mathrm{x}$ and $\mathrm{z}$

\section{CONCLUSION}

In this paper, a singularity free inverse kinematic solution method of serial robot manipulators is implemented to the cooperative working of two industrial robot manipulators. This solution method is based on screw theory and quaternion algebra. Compared with other methods, screw theory methods just establish two coordinates, and its geometrical meaning is obvious. And also screw theory with dual-quaternion method is the most compact and efficient way to express screw displacement. As the complexity and the degrees of freedom of the system increase, screw theory with dual-quaternion method gives better results since these systems have much more singularity points, computational loads and complex geometrical structure. Therefore, the wider use of the screw theory based methods with dual-quaternion in robot kinematic studies has to be considered by robotics community.

In the future works, collision free path planning of multi arm robot systems should be studied by using this formulation method. In addition, velocity and dynamic analysis based on screw theory with quaternion algebra should be studied.

\section{REFERENCE}

[1] Buckingham, Rob, "Multi -arm Robots", Industrial Robot Volume 23 Number $1 \cdot 1996 \cdot$ pp. 16-20C MCB University Press · ISSN 0143-9X

[2] Edsinger, A.; Kemp, C. C., "Two Arms Are Better Than One: A Behavior Based Control System for Assistive Bimanual Manipulation”,
Lecture Notes in Control and Information Sciences, Springer Berlin Heidelberg, Volume 370/2008, ISBN 0170-8643

[3] Park, C.; Park, K., "Design and Kinematics Analysis of Dual Arm Robot Manipulator for Precision Assembly" The IEEE international conference on Industrial Informatics (INDIN 2008)

[4] Chiacchio, P.; Chiaverini, S. ; Siciliano, B., "Direct and inverse kinematics for coordinated motion tasks of a two-manipulator system", Journal of dynamic systems, measurement, and control, CODEN JDSMAA 1996, vol. 118, nº 4, pp. 691-697

[5] Konietschke, R.; Ortmaier, T.; Hagn, U.; Hirzinger, G.; Frumento, S.," Kinematic Design Optimization of an Actuated Carrier for the DLR Multi-Arm Surgical System", Intelligent Robots and Systems IEEE/RSJ 9-15 Oct. 2006 pp: $4381-4387$

[6] Zollner, R.; Asfour, T.; Dillmann, R.," Programming by demonstration: dual-arm manipulation tasks for humanoid robots", Intelligent Robots and Systems, 2004. (IROS 2004). Proceedings. 2004 IEEE/RSJ International Conference on Volume 1, Issue , 28 Sept.-2 Oct. 2004 pp: 479 - 484 vol.1

[7] Zivanovic, M. D. ; Vukobratovic, M. K., "Multi-Arm Cooperating Robots", Dynamics and Control Series: Intelligent Systems, Control and Automation: Science and Engineering, Vol. 30, 2006, XV, 288p., ISBN: 978-4020-4268

[8] Jazidie, A.; Tsuji, T.; Nagamachi, M.; Ito, K., "Dynamic Simulation of Multi-Arm Robots Using Appel's Method”, IFToMM-jc International Symposium on Theory of Machines and Mechanisms, pp.199-204, 1992.

[9] Hemami, A., "Kinematics of Two-Arm Robots", IEEE Journal of Robotics and Automation, vol. RA-2, no.4 Dec. 1986

[10] Zheng, Y.F.," Kinematics and dynamics of two industrial robots in assembly", Robotics and Automation, 1989. Proceedings., 1989 IEEE International Conference onVolume, Issue , May 1989 pp:1360 -1365

[11] Ball, R. S., "The Theory of Screws", Cambridge, U.K.: Cambridge Univ.Press, 1900.

[12] Z.Huang and YL.Yao "Extension of Usable Workspace of Rotational Axes in Robot Planing", Robotica (1999) volume 17, pp. 293-301. Printed in the United Kingdom (C) 1999 Cambridge University Press

[13] Yang, A.T.; Freudenstein, F., "Application of dual-number quaternions to the to the analysis of spatial mechanism", ASME Trans. J. Applied Mechanics, 300-306 (1964)

[14] Yang, A.T., "Displacement analysis of spatial five-link mechanism using (3 x 3) matirces with dual number elements", ASME Trans. J. Engr. For Industry Feb.1969, 152-157

[15] M.Murray, Z.Li and S.S. Sastry, "A mathematical introduction to robotic manipulation," Boca Raton FL:CRC Press, 1994

[16] J. Funda and R. P. Paul, "A computational analysis of screw transformations in robotics," IEEE Trans. Robot. Automat., vol. 6, pp. 348-356,June 1990

[17] J. Funda, R. H. Taylor, and R. P. Paul, "On homogeneous transforms,quaternions, and computational efficiency," IEEE Trans. Robot. Automat., vol. 6, pp. 382-388, June 1990.

[18] E. Sariyildiz and H. Temeltas, "Solution of Inverse Kinematic Problem for Serial Robot Using Dual Quaterninons and Plücker Coordinates", Advanced intelligent mechatronics, 2009F

[19] E. Sariyildiz and H. Temeltas, "Solution of Inverse Kinematic Problem for Serial Robot Using Quaterninons", International Conference on Mechatronics and Automation, 2009

[20] H. Bruyninckx and J. D. Schutter , "Introduction toIntelligent Robotics" 7th edition 1October 2001

[21] W.R. Hamilton, Elements of Quaternions, Vol. I, \& II, New York, Chelsea, 1869.

[22] Qing Tan; Balchen, J.G. "General quaternion transformation resentation for roboticapplication”, International Conference on Volume , Issue , 17-20 Oct 1993 Page(s):319 - 324 vol.3

[23] J.M. Selig, Geometrical Fundamentals Of Robotics, Springer November $20042^{\text {nd }}$ edition, 9-20,25-34,41-49,81-90

[24] Konstantinos Daniilidis ,"Hand-Eye Calibration Using Dual Quaternions", The International Journal of Robotics Research Vol. 18, No. 3, March 1999, pp. 286-298,

[25] B. Paden, Kinematics and Control Robot Manipulators, PhD thesis Department of Electrical Engineering and Computer Science, University of California,Berkeley,1986

[26] T. Yue-sheng, X. Ai-ping "Extension of the Second Paden-Kahan Sub-problem and its' Application in the Inverse Kinematics of a Manipulator", 978-1-4244-1676-9/08 (C2008 IEEE) 